\title{
Point Form Electrodynamics and the Construction of Conserved Current Operators
}

\author{
W. H. Klink \\ Department of Physics and Astronomy \\ University of Iowa, Iowa City, Iowa, USA
}

December 10, 2018

\begin{abstract}
A general procedure for constructing conserved electromagnetic current operators is given. The four-momentum operator in point form relativistic quantum mechanics is written as the sum of hadronic, photon, and electromagnetic four-momentum operators, where the electromagnetic four-momentum operator is generated from a vertex operator, in which a conserved current operator is contracted with the four-vector potential operator. The current operator is the sum of free, dynamically determined and model dependent operators, the sum of the first two constructed so as to be conserved with respect to the hadronic interactions. It is shown that the dynamically determined operator carries charge. The model dependent operator is a many-body current operator, formed as the commutator of an antisymmetric operator with the hadronic four-momentum operator. It is shown that such an operator is conserved and does not renormalize the charge.
\end{abstract}

\section{Introduction}

A constantly recurring issue in few-body quantum mechanics concerns the construction of conserved electromagnetic current operators. One-body current operators, such as those constructed in the second paper in this series [1],

${ }^{1}$ PACS, 11.40.Dw, 13.40.Gp. 
are conserved when there are no hadronic interactions. But in the presence of hadronic interactions they are no longer conserved.

Such issues already arise in nonrelativistic quantum mechanics. Let $H$ be the hadronic Hamiltonian, $\rho(\vec{x})$ the charge density operator, and $\vec{J}(\vec{x})$ the current density operator. The electromagnetic charge and current operators in the Heisenberg representation are then given by

$$
\begin{aligned}
& \rho(t, \vec{x})=e^{i H t} \rho(\vec{x}) e^{-i H t} \\
& \vec{J}(t, \vec{x})=e^{i H t} \vec{J}(\vec{x}) e^{-i H t},
\end{aligned}
$$

and the continuity equation, $\partial \rho(t, \vec{x}) / \partial t+\nabla \cdot \vec{J}(t, \vec{x})=0$ can be written as the commutator equation,

$$
i[H, \rho(\vec{x})]+\nabla \cdot \vec{J}(\vec{x})=0 .
$$

If the charge density operator $\rho(\vec{x})$ is chosen to be a one-body operator and the interaction terms in $H$ do not commute with the charge density operator, the current density operator will contain not only one-body terms, but two (or more) body terms in order that the continuity equation be satisfied. But this does not fix the current density operator, since the continuity equation only involves the divergence of the current density operator. If the current density operator is decomposed into so-called parallel and perpendicular components, then only the parallel component is fixed by the continuity equation, and the perpendicular component is left unspecified, resulting in what are called model dependent currents [2].

It will be shown that the same situation holds in relativistic quantum mechanics, with the additional stipulation that, in the presence of hadronic interactions, there must always be two (or more)-body currents, in order to be relativistically covariant. Moreover, the decomposition of the current operator into two parts, one of which is divergenceless, is not relativistically invariant. In the point form of relativistic quantum mechanics, the form used in this series of papers, one-body current operators with the correct Lorentz and other transformation properties were given in the previous paper for arbitrary spin particles [四; but, in the presence of hadronic interactions, in order to satisfy current conservation, there must nonetheless be two or more body operators. This is most readily seen in the Breit frame, where the zero component of the four vector current operator gives the electric form factors, and the one and two components give the magnetic form factors. The third component in the Breit frame should be zero in order for current conservation 
to hold. But a direct calculation shows that this is not the case if there are only one-body currents [3]. Only if (implicit) two or more-body currents are included, will current conservation hold.

In quantum field theory it might seem that once the Lagrangian for the fields is given, the electromagnetic current operator is uniquely given. But even here there are problems having to do with satisfying the field equations. Let $\mathcal{L}$ be the Lagrangian consisting of polynomials in free field operators, written as $\mathcal{L}=\mathcal{L}_{\text {matter }}+\mathcal{L}_{\gamma}+\mathcal{L}_{\text {em }}$, where $\mathcal{L}_{\text {matter }}$ is the matter Lagrangian, for example for pions and nucleons, $\mathcal{L}_{\gamma}$ is the photon Lagrangian, and $\mathcal{L}_{e m}$ is the electromagnetic Lagrangian, in which the charged matter fields are coupled to the photon field. Usually the electromagnetic part of the Lagrangian is given through minimal substitution. Then Noether's theorem says that if $\mathcal{L}$ is invariant under some internal symmetry transformation, for example a constant phase transformation, there exists a quantity $J_{\mu}(x)$ which is conserved if the field equations are satisfied. But solving the field equations in the presence of interactions is very difficult, even for the electron-photon system, let alone for the pion-nucleon system to which the photon field must be coupled [4].

One of the goals of this paper is to show how to construct conserved electromagnetic current operators in the presence of hadronic interactions. The current operators will be the sum of one-body and many-body current operators, pieces of which will be the analogues of the model dependent and model independent non-relativistic currents. The one-body current operators have already been constructed in the second paper in this series; however, since they are not conserved with respect to the hadronic interactions, they will be modified by adding pieces that make them conserved with respect to the hadronic interactions. Such a modification is analogous to the model independent addition to the one-body currents in the non-relativistic case.

The construction of the analog of the model dependent currents in nonrelativistic quantum mechanics arises from the translational covariance properties of current operators. Many-body currents will be constructed that are conserved and carry zero charge, by analyzing, in section 3, the structure of point form electrodynamics. Such currents are given as the commutator of a field tensor with the hadronic four-momentum operator; the model dependence arises because the field tensor can be chosen from a wide variety of operators, including one-body current operators, as well as Feynman diagrams with external photon lines.

In section 2 the main features of point form relativistic quantum me- 
chanics are quickly reviewed, while in section 3 the ideas of point form electrodynamics are laid out. In particular it is shown that matrix elements of the current and electromagnetic field operators recover the classical Maxwell equations. Then in section 4 the analog of the model independent and in section 5 the analog of model dependent currents are constructed. The sum of free, model independent and model dependent currents gives the total current operator, which, when coupled to the the photon field, generates the electromagnetic vertex.

\section{Point Form Relativistic Quantum Mechan- ics}

In point form relativistic quantum mechanics all interaction are put in the four-momentum operator. The Lorentz generators contain no interactions so that the point form is manifestly Lorentz covariant. In fact the Lorentz generators will almost never be used; instead global Lorentz transformations are used to define the transformation properties of operators and states. In particular the Lorentz transformation properties of photons, worked out in reference [5], are needed for the electromagnetic vertex. If $U_{\Lambda}$ is a unitary operator representing the Lorentz transformation $\Lambda$, then the four-momentum operator must satisfy the following point form equations:

$$
\begin{aligned}
{\left[P_{\mu}, P_{\nu}\right] } & =0 \\
U_{\Lambda} P_{\mu} U_{\Lambda}^{-1} & =\left(\Lambda^{-1}\right)_{\mu}^{\nu} P_{\nu} .
\end{aligned}
$$

These equations are simply one way of writing the Poincaré commutation relations in which the relations of the four-momentum operators are emphasized. The mass operator is defined to be $M:=\sqrt{P \cdot P}$ and must have a nonnegative spectrum.

Since the four-momentum operators are the generators of space-time translations, they can be used to define the relativistic generalization of the time dependent Schrödinger equation,

$$
i \frac{\partial \Psi_{x}}{\partial x^{\mu}}=P_{\mu} \Psi_{x}
$$

where $\Psi_{x}$ is an element of the Hilbert space and $x\left(=x_{\mu}\right)$ is a space-time point. From Eq.3 it follows that $\Psi_{x}$ satisfies a generalized Klein-Gordan 
equation,

$$
\left(\frac{\partial}{\partial x^{\mu}} \frac{\partial}{\partial x_{\mu}}+M^{2}\right) \Psi_{x}=0,
$$

where $M$ is the mass operator. It is Eqs. 3,4 that take the place of equations of motion. If $P_{\mu}$ has no explicit space-time dependence, then Eq.3 can be written as an eigenvalue equation for the four-momentum operator:

$$
P_{\mu} \Psi=p_{\mu} \Psi \text {. }
$$

Assume now that $P_{\mu}$ is the sum of hadron, photon, and electromagnetic four-momentum operators, and satisfies Eqs.1,2. The hadron fourmomentum operator, $P_{\mu}(h)$, is assumed to be independent of photon creation and annihilation operators, while the photon four-momentum operator, $P_{\mu}(\gamma)$, is proportional to $c^{\dagger} c$ (see Eq.23, reference [5]). The main point of this paper is to show how to construct a conserved current operator for the electromagnetic vertex in the presence of hadronic interactions.

In particular, if the hadronic four-momentum operator is of BakamjianThomas form, which in the point form means that the four-momentum operator is the product of a mass and four-velocity operator, then, as will be shown in section 5, matrix elements of the model dependent current can be written as a commutator of the interacting mass operator with a freely chosen current operator.

\section{Point Form Electrodynamics}

The fundamental quantities in point form electrodynamics needed to construct many-body currents are the four-momentum operator, $P_{\mu}$, and the four-vector potential operator at the space-time point zero, $A_{\mu}(0)$ (which is always kinematic and defined via the photon creation and annihilation operators, see Eq.26, reference [5]). From these quantities the electromagnetic field tensor at the space-time point zero is defined as

$$
i F_{\mu \nu}(0):=\left[A_{\mu}(0), P_{\nu}\right]-\left[A_{\nu}(0), P_{\mu}\right]
$$

and is antisymmetric in $\mu$ and $\nu$. From this definition it follows that the electromagnetic field tensor at the space-time point $x$ satisfies the usual relationship with the four-vector potential:

$$
i F_{\mu \nu}(x):=i e^{i P \cdot x} F_{\mu \nu}(0) e^{-i P \cdot x}
$$




$$
\begin{aligned}
& =\left[A_{\mu}(x), P_{\nu}\right]-\left[A_{\nu}(x), P_{\mu}\right] \\
& =i\left(\frac{\partial A_{\mu}(x)}{\partial x^{\nu}}-\frac{\partial A_{\nu}}{\partial x^{\mu}}\right),
\end{aligned}
$$

where $A_{\mu}(x)=e^{i P \cdot x} A_{\mu}(0) e^{-i P \cdot x} . \quad A_{\mu}(0)$ is a linear combination of photon creation and annihilation operators and hence acts only on the photon Fock space. The correct Lorentz transformation properties of all the field quantities follow from the Lorentz properties of operators at the space-time point zero.

A electromagnetic current operator at the space-time point zero can then be defined as

$$
i J_{\mu}(0):=\left[F_{\mu \nu}(0), P^{\nu}\right],
$$

from which it follows that

$$
\begin{aligned}
i J_{\mu}(x) & =i e^{i P \cdot x} J_{\mu}(0) e^{-i P \cdot x} \\
& =e^{i P \cdot x}\left[F_{\mu \nu}(0), P^{\nu}\right] e^{-i P \cdot x} \\
& =i \frac{\partial F_{\mu \nu}(x)}{\partial x_{\nu}}
\end{aligned}
$$

Such a current is always conserved. This follows directly from the antisymmetry of $F_{\mu \nu}(x)$ and Eq.12, but it is worthwhile deriving current conservation as a commutator relation, namely $\left[P^{\mu}, J_{\mu}(0)\right]=0$, since this form of current conservation will be used in the following section.

Now, $\partial J_{\mu}(x) / \partial x_{\mu}=0$ is equivalent to $\left[P^{\mu}, J_{\mu}(0)\right]=0$. But

$$
\begin{aligned}
i\left[P^{\mu}, J_{\mu}(0)\right] & =\left[P^{\mu},\left[F_{\mu \nu}(0), P^{\nu}\right]\right] \\
& =-\left[F_{\mu \nu}(0),\left[P^{\nu}, P^{\mu}\right]\right]-\left[P^{\nu},\left[P^{\mu}, F_{\mu \nu}(0)\right]\right] \\
& =\left[P^{\nu},\left[F_{\mu \nu}(0), P^{\mu}\right]\right] \\
& =-\left[P^{\mu},\left[F_{\mu \nu}(0), P^{\nu}\right]\right] \\
& =0 .
\end{aligned}
$$

To arrive at this result the Jacobi identity for three operators was used, as well as the antisymmetry of $F_{\mu \nu}(0)$ and the point form Eq.1. Thus, any current operator defined by Eq.10 is conserved if the electromagnetic field tensor is antisymmetric and the four-momentum operators commute with one another. 
Eq.12 turns around the usual relationship between field and current. In classical electrodynamics, Eq.12 is usually interpreted as a differential equation for the field in the presence of specified currents. In this paper Eq.10 will define a many-body current, given $F$ and the four-momentum operator. Moreover, such a current is not only conserved, but also carries zero charge. That is, matrix elements of the charge operator on eigenstates of the four-momentum operator are zero:

$$
\begin{aligned}
i<p^{\prime} \ldots|\hat{Q}| p \ldots> & =i \int d^{3} x<p^{\prime} \ldots\left|J_{\mu=0}(\vec{x})\right| p \ldots> \\
& =i \int d^{3} x<p^{\prime} \ldots\left|e^{i \vec{P} \cdot \vec{x}} J_{\mu=0}(0) e^{-i \vec{P} \cdot \vec{x}}\right| p \ldots> \\
& =\int d^{3} x e^{i(\vec{p}-\vec{p}) \cdot \vec{x}}<p^{\prime} \ldots\left|\left[F_{0 \nu}(0), P^{\nu}\right]\right| p \ldots> \\
& =\delta^{3}(\vec{p}-\vec{p})\left(p-p^{\prime}\right)^{\nu}<p^{\prime} \ldots\left|F_{0 \nu}(0)\right| p \ldots> \\
& =0 ;
\end{aligned}
$$

here $\mid p \ldots>$ is an eigenstate of the four-momentum operator (the dots indicate other quantum numbers not needed in the derivation) and it is assumed that the two eigenstates have the same mass.

Because fields, in general, are not local, gauge transformations are defined by adding an operator (rather than c-number) to $A_{\mu}(x)$ :

$$
A_{\mu}^{\prime}(0):=A_{\mu}(0)+i\left[P_{\mu}, \chi(0)\right],
$$

where $\chi(0)$ is a Lorentz scalar operator. If $\chi(0)$ is space-time translated to $\chi(x)$, it follows that

$$
\begin{aligned}
A_{\mu}^{\prime}(x) & =A_{\mu}(x)+\frac{\partial \chi(x)}{\partial x^{\mu}} \\
i F_{\mu \nu}^{\prime}(0): & =\left[A_{\mu}^{\prime}(0), P_{\nu}\right]-\left[A_{\nu}^{\prime}(0), P_{\mu}\right] \\
& =i F_{\mu \nu}(0),
\end{aligned}
$$

so that the electromagnetic field tensor is gauge invariant. Since the gauge transformation defined in Eq.16 involves a scalar operator rather than a c-number function, as is the case for local fields, the link to gauge transformation in terms of photon creation and annihilation operators is different than that given in reference [5]. The link is given by writing $\chi(0)$ in terms of momentum dependent operators:

$$
\chi(0)=\int \frac{d^{3} k}{k_{0}}\left(\tilde{c}(k)+\tilde{c}(k)^{\dagger}\right),
$$


where $\tilde{c}(k)^{\dagger}$ is the adjoint of the operator $\tilde{c}(k)$ and guarantees that $\chi(0)$ is hermitian. Using the results of reference [0], Eq.31, where $A_{\mu}(0)\left(A_{\mu}^{\prime}(0)\right)$ is expanded in terms of photon creation and annihilation operators, and keeping only the annihilation operator parts, Eq.16 becomes

$$
\begin{aligned}
-\epsilon_{\mu}(k, \alpha) g_{\alpha, \alpha}\left(c^{\prime}(k, \alpha)-c(k, \alpha)\right) & =i\left[P_{\mu}, \tilde{c}(k)\right] \\
& =-k_{\mu} f(k) I . \\
{\left[P_{\mu}, \tilde{c}(k)\right] } & =i k_{\mu} f(k) I
\end{aligned}
$$

Since the four-momentum operator $P_{\mu}$ contains all the dynamics, Eq.18 implies that the gauge operator $\tilde{a}(k)$ also is dynamical, while the c-number gauge transformation $f(k)$ given in Eq.31 of reference [5] remains kinematical. $\epsilon_{\mu}(k, \alpha)$ is the polarization vector.

To conclude this section Maxwell's equations are shown to be expectation values of the operator relations. Thus,

$$
\begin{aligned}
<F_{\mu \nu}(x)> & =\left(\Psi_{0}, e^{i P \cdot x} F_{\mu \nu}(0) e^{-i P \cdot x} \Psi_{0}\right) \\
& =\left(\Psi_{x}, F_{\mu \nu}(0) \Psi_{x}\right) \\
\frac{\partial}{\partial x_{\nu}}<F_{\mu \nu}(x)> & =\left(\Psi_{x}, J_{\mu}(0) \Psi_{x}\right) \\
& =<J_{\mu}(x)> \\
<F_{\mu \nu}(x)> & =\frac{\partial}{\partial x^{\nu}}<A_{\mu}(x)>-\frac{\partial}{\partial x^{\mu}}<A_{\nu}(x)>,
\end{aligned}
$$

where use has been made of the relativistic Schrödinger equation, Eq.3 and $\Psi_{0}$ is a wavefunction at the space-time point zero. Now in general $\frac{\partial}{\partial x_{\mu}}<A_{\mu}(x)>\neq 0$. But, just as in classical electrodynamics, where one may make a gauge transformation so that the four divergence of the vector potential is zero, so too the operator $\chi(0)$ can be chosen so that the delambertian of expectation values of $\chi(x)$ produces a four-divergence of expectation values of the four-vector potential being zero. The point is that for any four-momentum operator satisfying the point form equations, Eqs.1,2, an operator gauge transformation, Eq.16 results in a gauge invariant field operator, $F_{\mu \nu}(0)$, as seen in Eq.18, and a gauge invariant current operator. 


\section{Dynamically Determined Many-Body Cur- rents}

The goal of this paper is to construct conserved electromagnetic current operators on the hadronic Hilbert space as the sum of one-body current operators defined in the previous paper [1] , and a many-body current operator,

$$
J_{\mu}(0):=J_{\mu}^{1}(0)+J_{\mu}^{\text {many-body }}(0),
$$

itself the sum of dynamically determined and model dependent pieces. In this section the dynamically determined currents will be built from the free current operators constructed in reference [1]. As previously noted, such operators are conserved for the free hadronic four-momentum operator, but not in the presence of hadronic interactions. This can be seen most clearly by writing translational covariance as

$$
\begin{aligned}
J_{\mu}(x) & =e^{i P \cdot x} J_{\mu}(0) e^{-i P \cdot x} \\
{\left[P_{\mu}, J_{\nu}(x)\right] } & =-i \frac{\partial J_{\nu}(x)}{\partial x^{\mu}}
\end{aligned}
$$

$J_{\nu}(x)$ is clearly conserved if $\left[P^{\mu}, J_{\mu}(0)\right]=0$. This is the case if the fourmomentum operator is the free four-momentum operator and the current is a one-body current. However, if the (hadronic) four-momentum operator is the sum of free and interacting four-momentum operators, $P^{\mu}(h)=P^{\mu}(f r)+$ $P^{\mu}(I)$, (satisfying Eqs.1,2), current conservation will not hold unless a term (denoted by "DD" for dynamically determined) is added to the one-body current operator:

$$
\left[P^{\mu}(f r)+P^{\mu}(I), J_{\mu}^{1}(0)+J_{\mu}^{D D}(0)\right]=0 .
$$

If the interacting four-momentum operator, $P^{\mu}(I)$ does not commute with the one-body current operator, $J_{\mu}^{1}(0)$, there must be additional (dynamically determined) currents present in order to satisfy current conservation, exactly as in the non-relativistic case.

Making use of the fact that the one-body current operator is conserved with respect to the free four-momentum operator, $\left[P^{\mu}(f r), J_{\mu}^{1}(0)\right]=0$, and taking eigenfunctions of the hadronic four-momentum operator to construct current operator matrix elements, Eq.27 becomes

$$
Q^{\mu}<p^{\prime}\left|J_{\mu}^{D D}(0)\right| p>=-<p^{\prime}\left|\left[P^{\mu}(I), J_{\mu}^{1}(0)\right]\right| p>
$$


where $Q^{\mu}$ is the four-vector momentum transfer, $Q^{\mu}=\left(p^{\prime}-p\right)^{\mu}$.

A general solution to Eq.28 is given by

$$
\begin{aligned}
<p^{\prime}\left|J_{\mu}^{D D}(0)\right| p> & =-\frac{\left(Q_{\mu}+Q_{\mu}^{\perp}\right)}{Q^{2}}<p^{\prime}\left|\left[P^{\nu}(I), J_{\nu}^{1}(0)\right]\right| p> \\
& =-\frac{\left(Q_{\mu}+Q_{\mu}^{\perp}\right)}{Q^{2}} Q^{\nu}<p^{\prime}\left|J_{\nu}^{1}(0)\right| p>;
\end{aligned}
$$

here $Q^{\perp}$ is a four-vector perpendicular to $Q, Q \cdot Q^{\perp}=Q^{\mu} Q_{\mu}^{\perp}=0$, and is determined by the requirement that there should be no pole at $Q^{2}=0$. As can be seen from Eq.29, if the interacting four-momentum operator is zero, the dynamically determined current operator also is zero.

To see the effects of adding a dynamically determined many-body current operator to the free current operator to ensure current conservation, it is useful to go to a (generalized) Breit frame, where $p^{\prime}(s t)=m^{\prime}(\operatorname{ch} \alpha, 0,0$, sh $\alpha)$, $p(s t)=m(\operatorname{ch} \alpha, 0,0,-s h \alpha)$, and $Q(s t)=\left(\left(m^{\prime}-m\right) \operatorname{ch} \alpha, 0,0,\left(m^{\prime}+m\right) s h \alpha\right)$. The appendix shows that if $Q^{\perp}=-\left(\left(m^{\prime}+m\right) \operatorname{sh} \alpha, 0,0,\left(m^{\prime}-m\right) c h \alpha\right)$ there will be no pole at $Q^{2}=0$. Note that if the masses of the initial and final state are the same $\left(m^{\prime}=m\right)$, the four-vector momentum transfer is the usual one, with only the third component nonzero. Then the third component of the total current in the Breit frame is zero, because the contribution from the one-body current matrix element is exactly balanced by the dynamically determined current, as seen in Eq.30.

More generally the current operator is

$$
\begin{aligned}
J_{\mu}(0) & =J_{\mu}^{1}(0)+J_{\mu}^{D D}(0) \\
<p^{\prime}\left|J_{\mu}(0)\right| p> & =<p^{\prime}\left|J_{\mu}^{1}(0)\right| p>-\frac{Q_{\mu}+Q_{\mu}^{\perp}}{Q^{2}} Q^{\nu}<p^{\prime}\left|J_{\nu}^{1}(0)\right| p>
\end{aligned}
$$

and from Eq.32 it follows that $J_{\mu}(0)$ is conserved, $Q^{\mu}<p^{\prime}\left|J_{\mu}(0)\right| p>=0$.

Moreover the form of $J_{\mu}^{D D}(0)$ in Eq.30 implies it carries charge. Using Eq.14 for the charge operator, the relevant matrix element to be evaluated is $<p\left|J_{\mu=0}^{D D}(0)\right| p>$; since such matrix elements in the point form are Lorentz covariant, it is most convenient to use the Breit frame and set $\alpha=0$. Then

$$
\begin{aligned}
<p^{\prime}(s t)\left|J_{\mu=o}^{D D}(0)\right| p(s t)>\left.\right|_{\alpha=0}= & -\frac{\left(Q(s t)+Q^{\perp}(s t)\right)_{\mu=0}}{Q^{2}} \\
& Q^{\nu}<p^{\prime}(s t)\left|J_{\nu}^{1}(0)\right| p(s t)>\left.\right|_{\alpha=0} \\
= & -<p^{\prime}(s t)\left|J_{\nu=3}^{1}(0)\right| p(s t)>\left.\right|_{\alpha=0},
\end{aligned}
$$


so that the total charge content is given by

$$
<p^{\prime}(s t)\left|J_{\mu=0}(0)\right| p(s t)>=<p^{\prime}(s t)\left|J_{\mu=0}^{1}(0)-J_{\mu=3}^{1}(0)\right| p(s t)>
$$

for $\alpha=0$.

To see the implications for the total charge as a sum of constituent charges consider the simple model in which a spinless bound state of mass m is made from $\mathrm{n}$ spinless constituents of mass $m_{c}$. The goal is to compute the onebody matrix elements in Eq.35 for $\mu=0$ and $\mu=3$, by assuming that the current matrix element of the first constituent has the form $<p_{1}^{\prime}\left|J_{\mu}(0)\right| p_{1}>=$ $\left(p_{1}^{\prime}+p_{1}\right)_{\mu} f_{1}\left(\left(p_{1}^{\prime}-p_{1}\right)^{2}\right)$, with $f_{1}()$ an arbitrary constituent form factor, whose value at 0 is the charge of the first constituent. The other constituents will have similar current matrix elements.

To carry out such a computation it is convenient to switch from single particle constituent variables, $p_{i}, i=1 \ldots n$ to internal variables, $k_{i}$, with $\sum \overrightarrow{k_{i}}=\overrightarrow{0}$; the connection is given by $p_{i}=B(v) k_{i}$, where $B(v)$ is a boost Lorentz transformation taking the four-vector $(1,0,0,0)$ to the four-velocity $v\left(v \cdot v=v^{\mu} v_{\mu}=1\right)$. The Jacobian $\mathcal{J}$ which converts the relativistic measure $\prod \frac{d^{3} p_{i}}{2 E_{i}}$ to a measure in velocity and internal momentum variables is given by

$$
\begin{aligned}
\prod \frac{d^{3} p_{i}}{2 E_{i}} & =\frac{d^{3} v}{v_{0}} \mathcal{J} \prod^{\prime} d^{3} k_{i} \\
\mathcal{J} & =\frac{M_{0}}{\prod 2 \omega_{i}},
\end{aligned}
$$

where the prime on the product in Eq.36 indicates a product over $n-1$ variables rather than $n$ variables, $M_{0}$ is the total n-particle mass, $M_{0}=$ $\sqrt{\sum p_{i} \cdot \sum p_{i}}=\sum \omega_{i}$, and $\omega_{i}$ is the energy of the $i^{t h}$ constituent, $\omega_{i}=$ $\sqrt{m_{c}^{2}+\vec{k}_{i}^{2}}$. If $\Psi\left(\overrightarrow{k_{i}}\right)$ is the bound state wavefunction for the particle of mass $m$, normalized to $\|\Psi\|^{2}=\int \mathcal{J} \prod^{\prime} d^{3} k_{i}\left|\Psi\left(\overrightarrow{k_{i}}\right)\right|^{2}=1$, then the desired current matrix element can be written as

$$
\begin{aligned}
<p^{\prime}(s t)\left|J_{\mu}^{1}(0)\right| p(s t)>= & \int \mathcal{J}^{\prime} \prod d^{3} k_{i}^{\prime} \mathcal{J} \prod^{\prime} d^{3} k_{i} \Psi^{*}\left(k_{i}^{\prime}\right) \\
& <v^{\prime}(s t) k_{i}^{\prime}\left|J_{\mu}^{1}(0)\right| v(s t) k_{i}>\Psi\left(k_{i}\right) ; \\
<v^{\prime}(s t) k_{i}\left|J_{\mu}^{1}(0)\right| v(s t) k_{i}>= & \prod^{\prime} 2 \omega_{i} \delta^{3}\left(k_{i}^{\prime}-B^{-1}\left(v^{\prime}(s t)\right) B(v(s t)) k_{i}\right) \\
& \left(p_{1}^{\prime}+p_{1}\right)_{\mu} f_{1}\left(\left(p_{1}^{\prime}-p_{1}\right)^{2}\right) \mathcal{F} .
\end{aligned}
$$


Eq.39 is the matrix element when only constituent 1 has been struck; to get the total charge similar matrix elements for all the other constituents must be added to Eq.39. The factor $\mathcal{F}$ is needed to ensure that for $\alpha=0$ the current matrix element, Eq.38 gives the correct total charge. That is, when $\alpha=0$, $v^{\prime}(s t)=v(s t)=(1,0,0,0)$ and the current matrix element for constituent 1 being struck is given by

$$
\begin{aligned}
<v^{\prime}(s t) k_{i}\left|J_{\mu}^{1}(0)\right| v(s t) k_{i}>\left.\right|_{\alpha=0}= & f_{1}(0) \int \mathcal{J} \prod^{\prime} d^{3} k_{i}\left|\Psi\left(k_{i}\right)\right|^{2} \\
& \frac{M_{0}}{2 \omega_{1}} 2\left(k_{1}\right)_{\mu} \mathcal{F} .
\end{aligned}
$$

The requirement on the charge operator, Eq.35, is that the charge of the bound state particle should equal the sum of the constituent charges. If there were no dynamically determined currents, Eq. 35 would say that only the zero component of the one-body current matrix element contribute; in that case, $\mathcal{F}=1 / M_{0}$. However, the current in Eq.35 is the sum of one-body and dynamically determined currents, which means the factor must include both

the zero and third component; in that case $\mathcal{F}=\frac{\omega_{1}}{M_{0}\left(\omega_{1}-k_{1 z}\right)}$. Thus it is clear that the dynamically determined current carries charge and must be taken into account in order that the total charge be the sum of the constituent charges.

\section{Model Dependent Currents}

The modification of $J_{\mu}^{1}$ to $J_{\mu}=J_{\mu}^{1}+J_{\mu}^{D D}$ corresponds to the addition of a dynamically determined term to the free current operator, analogous to the model independent current in non-relativistic quantum mechanics. The goal of this section is to construct the analogue of model dependent currents in nonrelativistic quantum mechanics, by using the current operators defined in section 3. Such currents, it will be recalled, are defined as the commutator of an antisymmetric operator with the hadronic four-momentum operator (see Eq.10); though the antisymmetric operator in Eq.10 is the electromagnetic field tensor, the only property used in showing that the current operator is conserved is that the operator in the commutator be antisymmetric. Moreover Eq.14 shows that the current also carries no charge. These results are the point form analogue of the classical electrodynamics result, namely that 
it is always possible to add to the current a term of the form $\frac{\partial A^{\mu \nu}(x)}{\partial x^{\nu}}$ (where $A^{\mu \nu}(x)$ is an antisymmetric field) that is conserved and carries no charge [6].

When $F_{\mu \nu}$ is replaced by an arbitrary antisymmetric operator, it should satisfy a cluster requirement, that when the hadronic interactions are zero, $A_{\mu \nu}$ should also be zero. That is, there should be no model dependent currents when the interacting hadronic mass operator is zero. Such a requirement is readily satisfied by writing the field tensor as

$$
i A_{\mu \nu}:=\left[I_{\mu}, P_{\nu}(I)\right]-\left[I_{\nu}, P_{\mu}(I)\right]
$$

where $P_{\mu}(I)$ is the interacting hadronic four-momentum operator and $I_{\mu}$ is any (current) operator that transforms as a four-vector. It need not be conserved and can carry charge; the model dependent current operator that it generates will of course be conserved and carry no charge. Examples of $I_{\mu}$ are the original one-body current operator, with or without additional form factors, as well as any Feynman diagram with an external photon line, viewed as the kernel of a current operator.

Matrix elements of the model dependent (MD) current operator are given by

$$
\begin{aligned}
<p^{\prime} \ldots\left|J_{\mu}^{M D}\right| p \ldots>= & Q^{\nu}<p^{\prime} \ldots\left|\left[I_{\mu}, V_{\nu} M_{I}\right]\right| p \ldots> \\
& -Q^{\nu}<p^{\prime} \ldots\left|\left[I_{\nu}, V_{\mu} M_{I}\right]\right| p \ldots>
\end{aligned}
$$

which can be simplified by going to the Breit frame:

$$
\begin{aligned}
<p^{\prime}(s t) \ldots\left|J_{\mu}^{M D}\right| p(s t) \ldots>= & -2 m \operatorname{sh}^{2} \alpha<p^{\prime}(s t) \ldots\left|\left[I_{\mu}, M_{I}\right]_{+}\right| p(s t) \ldots> \\
& +2 m \operatorname{sh} \alpha c h \alpha \\
& <p^{\prime}(s t) \ldots\left|\left[M_{I}, I_{3}\right]\right| p(s t) \ldots>\delta_{\mu 0}, \quad \text { (4) }
\end{aligned}
$$

for $\mu=0,1,2$; for $\mu=3$ the matrix element is zero. To see the effects of model dependent currents on form factors requires choosing a current $I_{\mu}$ and then calculating integrals of the form given in Eq.38; this will not be done here.

\section{Conclusion}

In this paper a general procedure for constructing conserved current operators has been given. The total current operator is written as the sum of three 
operators, a free current operator plus two many-body operators. The free current operator must be modified because it is not conserved with respect to the hadronic interactions. As shown in section 4 the procedure for modifying it is dynamically determined in the sense that no new operators are invoked to make it conserved. The current operator of section 5 is, on the other hand, highly model dependent, in that many different operators can be chosen to generate the field tensor in Eq.41, which then generates the model dependent conserved current.

Though the dynamically determined current follows from current conservation, as is also the case in nonrelativistic quantum mechanics, the form it takes (see Eq.30 ) has been used in the past to attain current conservation phenomenologically [7]. What has been shown in section 4 is that such a correction is incomplete, in that another term, the $Q_{\mu}^{\perp}$ in Eq.30 is also needed, to guarantee that there are no poles in the dynamically determined current. This in turn leads to the possibility that the dynamically determined current carries charge, which was investigated by looking at a simple bound state model. This model shows that indeed the dynamically determined current carries charge, and must be taken into account, in order that the total charge equal the sum of the constituent charges.

The motivation for this work comes from calculations done for elastic deuteron [8] and nucleon form factors [9], which give very different results when compared to data. In both calculations the point form of relativistic quantum mechanics is used to compute matrix elements of free current operators in the Breit frame [3]. In the deuteron calculation the A structure function is below the data already at momentum transfers of $2 \mathrm{Gev}^{2}$. Clearly many-body currents are needed to get better agreement with data. On the other hand, using free current operators for constituent quarks with no anomalous magnetic moments or other internal structure, Breit frame matrix elements give very good agreement with data, except for some static properties of the nucleons.

In order to try to understand these results it is necessary to construct many-body currents that change the deuteron form factors, but not the nucleon form factors. These many-body currents should be conserved, but not renormalize the charge. It is exactly such many-body currents that have been constructed in this paper. Since the interacting hadronic mass operator has been chosen in such a way as to give wave functions whose eigenvalues are in good agreement with data, and the dynamically determined currents are fixed by the one-body currents, the only way in which different model cur- 
rents can be introduced to see how they influence the deuteron and nucleon wave functions is through the model dependent currents, given in Eq.41 by the current $I_{\mu}$. Actual calculations of dynamically determined and model dependent currents for nucleon and deuteron form factors remain to be carried out.

\section{7 appendix}

In this appendix matrix elements of the dynamically determined current operator are analyzed to see if there is a pole at $Q^{2}=0$ when the initial and final four-momenta may have different masses (inelastic form factors). In such a situation there is no longer a unique definition of a Breit frame, but the generalization chosen here reduces to the usual definition when the initial and final masses are the same. From Eq.30 the matrix elements of the dynamically determined currents are

$$
<p^{\prime} \ldots\left|J_{\mu}^{D D}\right| p \ldots>=-\frac{\left(Q_{\mu}+Q_{\mu}^{\perp}\right) Q^{\nu}}{Q^{2}}<p^{\prime} \ldots\left|J_{\nu}^{1}\right| p \ldots>,
$$

with $Q=p^{\prime}-p$. If $Q \cdot Q^{\perp}=0$, then the sum of one-body plus dynamically determined current operators is conserved. Since the initial and final masses are no longer equal, the question is whether $Q^{\perp}$ can be chosen in such a way to ensure that there is no pole at zero momentum transfer [7].

To see how this is possible, choose a generalized Breit frame with $p^{\prime}(s t)=$ $m^{\prime}(\operatorname{ch} \alpha, 0,0, s h \alpha)$ and $p(s t)=m(\operatorname{ch} \alpha, 0,0,-s h \alpha)$; this reduces to the usual definition of a Breit frame when the two masses are equal. With these standard momenta, the momentum transfer squared is $Q^{2}(s t)=\left(m^{\prime}-m\right)^{2}-$ $4 m^{\prime} m s h^{2} \alpha$ and is zero when $s h \alpha=\frac{m^{\prime}-m}{2 \sqrt{m^{\prime} m}}$, which reduces to the elastic case when the masses are the same.

The goal now is to choose $Q^{\perp}(s t)$ so that $Q(s t) \cdot Q^{\perp}(s t)=0$ and there is no pole at $Q^{2}(s t)=0[\mathbb{8}]$. The condition that $Q(s t) \cdot Q^{\perp}(s t)=0$ means that

$$
\begin{aligned}
\left(Q+Q^{\perp}\right)(s t)= & \left(\left(m^{\prime}-m\right) \operatorname{ch} \alpha, 0,0,\left(m^{\prime}+m\right) \operatorname{sh} \alpha\right) \\
& +a\left(\left(m^{\prime}+m\right) \operatorname{sh} \alpha, 0,0,\left(m^{\prime}-m\right) \operatorname{ch} \alpha\right)
\end{aligned}
$$

where $a$ is a constant to be chosen so that $\left(Q+Q^{\perp}\right)(s t)=0$ at $Q^{2}(s t)=0$. Write $\alpha=\alpha_{0}$ at $Q^{2}(s t)=0$. Then $s h \alpha_{0}=\frac{m^{\prime}-m}{2 \sqrt{m^{\prime} m}}, c h \alpha_{0}=\frac{m^{\prime}+m}{2 \sqrt{m^{\prime} m}}$, and if 
$a=-1, Q+Q^{\perp}(s t)=0$ at $\alpha=\alpha_{0}$; thus the numerator and denominator are both zero at $Q^{2}(s t)=0\left(\alpha=\alpha_{0}\right)$. Moreover, if the numerator and denominator are both expanded about $\alpha=\alpha_{0}$, it is readily checked that the ratio is finite for $m^{\prime} \neq m$. The final result is that if

$$
\left(Q+Q^{\perp}\right)(s t)=\left[\left(m^{\prime}-m\right) \operatorname{ch} \alpha-\left(m^{\prime}+m\right) \operatorname{sh} \alpha\right](1,0,0,-1)
$$

the modified current in Eq.30 is conserved and has no pole at $Q^{2}=0$.

\section{References}

[1] W. H. Klink, "Local Current Operators for Arbitrary Spin Particles", submitted.

[2] see for example, D. O. Riska, Phys. Rep. 181 (1989) 207.

[3] W. H. Klink, Phys. Rev. C58 (1998) 3587.

[4] See for example, S. S. Schweber, An Introduction to Relativistic Quantum Field Theory (Harper and Row, New York,USA, 1962).

[5] W. H. Klink, "Point Form Electrodynamics and the Gupta-Bleuler Formalism", submitted.

[6] see for example, P Ramond, Field Theory, A Modern Primer (The Benjamin/Cummings Publishing Company, Inc., Reading, Massachusetts, USA, 1981), page 39 .

[7] see for example, J. Friar, Nucl. Phys. A264 (1976) 455.

[8] T. Allen, W. Klink, W. Polyzou, Phys. Rev. C63 (2001) 034002.

[9] R. F. Wagenbrunn, S. Boffi, W. Klink, W. Plessas, M. Radici, Phys. Lett. B511 (2001)33; B516 (2001) 183. 